\title{
Estrutura de cardumes da sardinha-verdadeira, Sardinella brasiliensis (Steindachner, 1879), no estado do Rio de Janeiro (Brasil)
}

\author{
Melquíades Pinto Paiva ${ }^{1,2}$ \\ Ana Paula da Costa Falcão ${ }^{1}$
}

\begin{abstract}
Schooling structure of Brazilian-sardine, Sardinella brasiliensis (Steindachner), in the State of Rio de Janeiro (Brazil). This paper deals with the schooling structure of Brazilian-sardine, Sardinella brasiliensis (Steindachner, 1879), in coastal waters of Rio de Janeiro State (Brazil). Samples were obtained from schools caught by commercial fishery in the area of Angra dos Reis $\left(23^{\circ}-24^{\circ} \mathrm{S}, 44^{\circ}-45^{\circ} \mathrm{W}\right)$, from autumn/1999 to summer/2000, comprising 2,278 fishes. In average, males had total lengths and weights lower than those of females, with major differences in weights. Seasonal fluctuations of average total weights are associated with the annual reproduction cycle and condition factor, mainly in females. Sex-ratio was around 1: 1 , except in winter, when females were in majority. Mature males and females were found all along the year, with a peak in the spring-summer, showing a long reproduction period of successive spawning of reproducers in a same school, since individual spawning is complete. Total lengths when $50 \%$ of fishes are mature differ between males $(18.2 \mathrm{~cm})$ and females $(19.2 \mathrm{~cm})$. The schools can be considered as spawning schools, as they were found in a spawning ground, and consisted almost exclusively of adults, with a long annual reproduction period.

KEY WORDS. Brazilian-sardine, Sardinella brasiliensis, schooling structure, Rio de Janeiro, Southeast Brazil
\end{abstract}

A sardinha-verdadeira, Sardinella brasiliensis (Steindachner, 1879), é endêmica da costa brasileira, geograficamente isolada das demais espécies do gênero, no Oceano Atlântico. Distribui-se desde o cabo de São Tomé ( $22^{\circ} \mathrm{S}$, Rio de Janeiro) até o cabo de Santa Marta Grande $\left(29^{\circ} \mathrm{S}\right.$, Santa Catarina) (VALENTINI \& Cardoso 1991). É o mais importante recurso pesqueiro marinho do Brasil, em volume de produção, com desembarques concentrados nos estados do Rio de Janeiro, São Paulo e Santa Catarina (PAIVA 1997).

Ao longo da costa do estado do Rio de Janeiro, os cardumes da sardinha-verdadeira são mais freqüentes e maiores na baía da Ilha Grande e águas adjacentes (área de Angra dos Reis, entre $23-24^{\circ} \mathrm{S}$ e $44-45^{\circ} \mathrm{W}$ ); os maiores cardumes são encontrados no outono e os menores no inverno. Os cardumes ocorrem em águas superficiais, em locais com até $80 \mathrm{~m}$ de profundidade, com marcada redução de suas freqüências, à medida que aumenta a profundidade dos locais das pescarias (PAIVA \& MOTTA 2000).

1) Departamento de Biologia Marinha, Universidade Federal do Rio de Janeiro. Cidade Universitária, Ilha do Fundão, 21944-970 Rio de Janeiro, Rio de Janeiro, Brasil.

2) Pesquisador do Conselho Nacional de Desenvolvimento Científico e Tecnológico (CNPq). 
Este trabalho trata da estrutura de cardumes da sardinha-verdadeira, em águas costeiras do estado do Rio de Janeiro (Brasil), considerando as estações do ano, tamanhos e sexos dos indivíduos amostrados, correlacionando os resultados com o ciclo anual de sua reprodução.

\section{MATERIAL E MÉTODOS}

Foram retiradas amostras mensais dos desembarques comerciais da sardinha-verdadeira em Angra dos Reis, costa sul do estado do Rio de Janeiro, entre abril/1999 e março/2000.

Os dados gerais das 12 amostras estão apresentados na tabela I, segundo as estações: outono $=$ abril-junho, inverno $=$ julho-setembro, primavera $=$ outubro-dezembro e verão = janeiro-março.

Os dados foram agrupados segundo as estações do ano, sexos e classes de tamanho dos peixes, para conhecimento dos seguintes parâmetros: proporção sexual (sex-ratio), fator de condição (K), ocorrência de indivíduos maduros, composição dos cardumes (classes de tamanho/fases do ciclo vital) e relações entre comprimentos totais e pesos totais dos peixes.

A determinação dos sexos foi baseada em características macroscópicas das gônadas. Machos com testículos bem desenvolvidos, deixando fluir esperma por leve pressão, foram considerados maduros. As fêmeas maduras tiveram identificação com base na escala de maturidade proposta por ISAAC-NAHUM et al. (1983), quando encontradas no estádio Cf, isto é, ovários desenvolvidos com óvulos bem visíveis (maduro desovando).

$\mathrm{O}$ cálculo do fator de condição $(\mathrm{K})$ foi procedido conforme a seguinte equação, apresentada em VAZZOLER \& VAZZOLER (1965): $\mathbf{K}=\left(\mathbf{W}_{\mathbf{t}} / \mathbf{L}_{\mathbf{t}}{ }^{\mathbf{3}}\right) \times \mathbf{1 0}^{\mathbf{3}}$, onde $\left(\mathrm{W}_{\mathrm{t}}\right)$ peso total do peixe $(\mathrm{g})$ e $\left(\mathrm{L}_{\mathrm{t}}\right)$ comprimento total do peixe $(\mathrm{cm})$.

Foram amostrados 2.278 peixes, sendo 953 machos e 1.049 fêmeas; os restantes 276 eram muito jovens e/ou estavam com as gônadas danificadas, impedindo a identificação dos sexos. Os comprimentos totais variaram entre 13,7 e 28,0 $\mathrm{cm}$ e os pesos totais entre $21,7 \mathrm{e} 178,7 \mathrm{~g}$ (Tab. I).

\section{RESULTADOS E DISCUSSÃO}

No conjunto das amostras, as médias de comprimento e peso totais dos machos foram menores do que as das fêmeas, com diferença mais acentuada no tocante ao peso total; o mesmo aconteceu nas estações do ano, com exceção do inverno, quando houve um certo equilíbrio das médias; não foi observada acentuada variação das médias de comprimento total de machos e fêmeas, bem como dos sexos em conjunto, entre as estações; quanto ao peso total, as médias variaram muito entre as estações, tanto dos machos como das fêmeas, e de ambos os sexos em conjunto; os pesos médios dos machos mostraram tendência decrescente do outono à primavera, crescendo no verão e com máximo no outono; nas fêmeas, as médias do peso total foram maiores no outono e menores no inverno, com tendência crescente na primavera e verão (Tab. I). 
Tabela I. Dados gerais sobre as amostras de cardumes da sardinha-verdadeira, capturados pela pesca comercial na área de Angra dos Reis (Rio de Janeiro, Brasil), entre o outono/1999 e o verão/2000.

\begin{tabular}{|c|c|c|c|c|c|}
\hline Estaçōes e ano & Sexos & Individuos (n) & Amplitudes & Médias & Desvios padrão \\
\hline \multicolumn{6}{|l|}{ Comprimento total $(\mathrm{cm})$} \\
\hline \multirow[t]{3}{*}{ Outono } & Machos & 191 & $14,8-21,8$ & 19,7 & 1.1 \\
\hline & Fêmeas & 169 & $17,3-23,5$ & 20,4 & 1,5 \\
\hline & Ambos & 594 & $14,8-23,5$ & 18,8 & 2,1 \\
\hline \multirow[t]{3}{*}{ Inverno } & Machós & 240 & $15,6-23,2$ & 19,5 & 1,2 \\
\hline & Fêmeas & 331 & $13,7-28,0$ & 19,4 & 1,8 \\
\hline & Ambos & 611 & $13,7-28,0$ & 19,3 & 1,7 \\
\hline \multirow[t]{3}{*}{ Primavera } & Machos & 291 & $15,0-26,0$ & 18,5 & 1,2 \\
\hline & Fêmeas & 310 & $16,6-23,3$ & 19,2 & 1.3 \\
\hline & Ambos & 602 & $15,0-26,0$ & 18,9 & 1,3 \\
\hline \multirow[t]{3}{*}{ Outono } & Machos & 231 & $15,4-21,2$ & 18,4 & 1,5 \\
\hline & Fêmeas & 239 & $15,4-22,2$ & 19,2 & 1,7 \\
\hline & Ambos & 471 & $15,4-22,2$ & 18,8 & 1,7 \\
\hline \multirow[t]{3}{*}{ Ano } & Machos & 953 & $14,8-26,0$ & 18,6 & 1,6 \\
\hline & Fêmeas & 1.049 & $13,7-28,0$ & 19,5 & 1,7 \\
\hline & Ambos & 2.278 & $13,7-28,0$ & 18,9 & 1,7 \\
\hline \multicolumn{6}{|l|}{ Peso total (g) } \\
\hline \multirow[t]{3}{*}{ Outono } & Machos & 191 & $27,5-95,8$ & 65,2 & 11,9 \\
\hline & Fêmeas & 169 & $37,8-112,1$ & 74,8 & 14,4 \\
\hline & Ambos & 594 & $27,5-112,1$ & 60,7 & 17,4 \\
\hline \multirow[t]{3}{*}{ Inverno } & Machos & 240 & $28,2-102,3$ & 64,3 & 11,8 \\
\hline & Fêmeas & 331 & $21,7-176,9$ & 63,2 & 16,9 \\
\hline & Ambos & 611 & $21,7-176,9$ & 62,4 & 15,6 \\
\hline \multirow[t]{3}{*}{ Primavera } & Machos & 291 & $30,0-178,7$ & 58,8 & 10,4 \\
\hline & Fêmeas & 310 & $41,9-119,1$ & 65,9 & 13,4 \\
\hline & Ambos & 602 & $30,0-178,7$ & 62,5 & 12,6 \\
\hline \multirow[t]{3}{*}{ Outono } & Machos & 231 & $33,4-90,9$ & 62,9 & 12,1 \\
\hline & Fêmeas & 239 & $34,5-108,1$ & 70,5 & 14,0 \\
\hline & Ambos & 471 & $33,4-108,1$ & 66,7 & 13,6 \\
\hline \multirow[t]{3}{*}{ Ano } & Machos & 953 & $27,5-178,7$ & 59,4 & 13,0 \\
\hline & Fêmeas & 1.049 & $21,7-176,9$ & 67,7 & 15,4 \\
\hline & Ambos & 2.278 & $21,7-178,7$ & 62,9 & 15,1 \\
\hline
\end{tabular}

Observações: quando o número de peixes é maior do que a soma de machos e fêmeas, isto decorre da não identificação dos sexos de alguns indivíduos; em junho/1999 não houve determinação dos sexos dos peixes.

As amostras foram coletadas na área norte de desova da sardinha-verdadeira, situada entre a Ilha Grande (Rio de Janeiro) e Ubatuba (São Paulo), onde as desovas ocorrem na primavera e verão (VAZZOLER \& VAZZOLER 1965; VAZZOLER \& ROSSIWONGTSCHOWSKI 1976; MATSUURA 1977, 1989; ISAAC-NAHUM et al. 1983). As flutuações entre as médias estacionais do peso total da sardinha-verdadeira estão associadas ao ciclo anual de reprodução, principalmente nas fêmeas (Tab. I).

A proporção sexual situou-se próxima de 1:1, exceto no inverno, quando houve maioria das fêmeas (Tab. II). Segundo MATSUURA (1977), durante o período da desova da sardinha-verdadeira, a proporção sexual é aproximadamente de 1:1, o que está de acordo com os dados deste trabalho.

Quanto aos valores de K, tanto para os machos como para as fêmeas, eles estão relacionados com o ciclo anual de reprodução da sardinha-verdadeira, com valores mínimos no inverno e máximos no verão (Tab. II). 
Na área de Angra dos Reis, machos e fêmeas maduros da sardinha-verdadeira são encontrados durante todo o ano, mas no inverno existem menos peixes maduros, com máximos na primavera-verão (Tab. II).

Tabela II. Relação sexual, valores de K e ocorrência de peixes maduros, nas amostras de cardumes da sardinha-verdadeira, capturados pela pesca comercial na área de Angra dos Reis (Rio de Janeiro, Brasil), entre o outono/1999 e o verão/2000. (爻) Média, (s) desvio-padrão.

\begin{tabular}{|c|c|c|c|c|c|}
\hline \multirow{2}{*}{ Estações e ano } & \multirow{2}{*}{$\begin{array}{c}\text { Proporção } \\
\text { (machos:fêmeas) }\end{array}$} & \multicolumn{2}{|c|}{ Valores de $K(\bar{x} \pm s)$} & \multicolumn{2}{|c|}{ Peixes maduros (\%) } \\
\hline & & Machos & Fêmeas & Machos $(n=183)$ & Fêmeas $(n=208)$ \\
\hline Outono & $1,0: 0,9$ & $9,1 \pm 0,9$ & $8,8 \pm 0,7$ & 10,5 & 14,8 \\
\hline Inverno & $1,0: 1,4$ & $8,5 \pm 0,5$ & $8,4 \pm 0,6$ & 7,5 & 3,0 \\
\hline Primavera & $1,0: 1,1$ & $9,2 \pm 0,6$ & $9,2 \pm 0,6$ & 29,9 & 38,1 \\
\hline Verão & $1,0: 1,0$ & $10,1 \pm 1,1$ & $10,0 \pm 1,1$ & 25,1 & 23,0 \\
\hline Ano & $1,0: 1,1$ & $9,2 \pm 0,9$ & $9,1 \pm 0,9$ & 19,2 & 19,8 \\
\hline
\end{tabular}

Observação: os números de machos e fêmeas, por estações e no ano, estão na tabela I.

A sardinha-verdadeira tem um longo período de reprodução, com sucessivas desovas dos reprodutores do cardume, sendo a desova individual completa (VAzZOLER \& ROSSI-WONGTSCHOWSKI 1976). Nas amostras estudadas, existiam machos e fêmeas em diferentes estágios de desenvolvimento sexual, o que confirma as sucessivas desovas nos cardumes. A desova completa é uma das características dos peixes de desova pelágica, entre os quais estão as sardinhas (MOYLE \& CECH 1982).

Indivíduos maduros da sardinha-verdadeira tiveram comprimentos totais mínimos de $15 \mathrm{~cm}$ para os machos e $14 \mathrm{~cm}$ para as fêmeas (Tab. III). Os comprimentos totais correspondentes a $50 \%$ de peixes maduros foram diferentes entre os sexos: 18,2 e 19,2 cm, respectivamente, para machos e fêmeas (Fig. 1). Assim, quanto ao comprimento total, os machos são mais precoces do que as fêmeas.

Tabela III. Ocorrência de peixes maduros por classes de comprimento total, nas amostras de cardumes da sardinha-verdadeira, capturados pela pesca comercial na área de Angra dos Reis (Rio de Janeiro, Brasil), entre o outono/1999 e o verão/2000.

\begin{tabular}{|c|c|c|c|c|}
\hline \multirow{3}{*}{ Comprimento total $(\mathrm{cm})$} & \multicolumn{4}{|c|}{ Freqüências de peixes maduros (\%) } \\
\hline & \multicolumn{2}{|c|}{ Machos $(n=183)$} & \multicolumn{2}{|c|}{ Fêmeas $(n=208)$} \\
\hline & Simples & Acumuladas & Simples & Acumuladas \\
\hline 14 & - & - & 0,0 & 0,0 \\
\hline 15 & 0,0 & 0,0 & 0,0 & 0,0 \\
\hline 16 & 6,6 & 6,6 & 0,0 & 0,0 \\
\hline 17 & 22,3 & 28,9 & 9,7 & 9,7 \\
\hline 18 & 19,3 & 48,2 & 15,9 & 25,6 \\
\hline 19 & 17,3 & 65,5 & 20,3 & 45,9 \\
\hline 20 & 21,8 & 87,3 & 27,1 & 73,0 \\
\hline 21 & 9,1 & 96,4 & 18,4 & 91.4 \\
\hline 22 & 3,1 & 99,5 & 7,2 & 98,6 \\
\hline 23 & 0,0 & 99,5 & 1,4 & 100,0 \\
\hline 24 & - & 99,5 & - & 100,0 \\
\hline 25 & - & 99,5 & - & 100,0 \\
\hline 26 & 0,5 & 100,0 & - & 100,0 \\
\hline 27 & - & 100,0 & - & 100,0 \\
\hline 28 & - & 100,0 & 0,0 & 100,0 \\
\hline Total & 100,0 & - & 100,0 & - \\
\hline
\end{tabular}




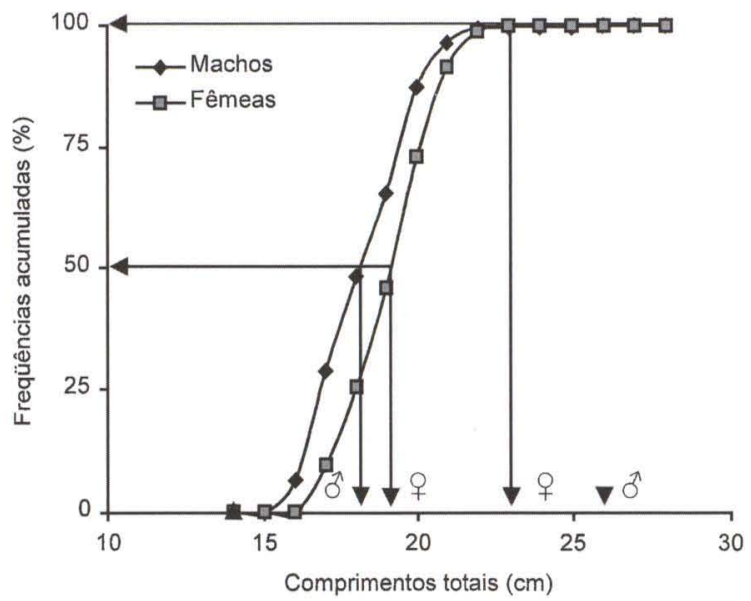

Fig. 1. Freqüências acumuladas ae machos e fêmeas maduros(as), em amostras de cardumes da sardinha-verdadeira, capturados pela pesca comercial, na área de Angra dos Reis (Rio de Janeiro, Brasil), entre o outono/1999 e o verão/2000.

Considerando os resultados aqui apresentados, é possível definir os seguintes agrupamentos, com respeito aos comprimentos totais da sardinha-verdadeira: jovens - com menos de $16 \mathrm{~cm}$ e $17 \mathrm{~cm}$ para machos e fêmeas, respectivamente; pequenos adultos - entre 16 e $18 \mathrm{~cm}$ nos machos e entre 17 e $19 \mathrm{~cm}$ nas fêmeas; grandes adultos - acima de $18 \mathrm{~cm}$ nos machos e de $19 \mathrm{~cm}$ nas fêmeas.

Quanto à composição dos cardumes da sardinha-verdadeira, por classes de comprimento total/agrupamentos do ciclo de vida (Tab. IV), pode-se observar o seguinte: nos machos - os jovens foram representados por $0,0 \%$ dos indivíduos, pequenos adultos por $24,4 \%$ e grandes adultos por $75,6 \%$; nas fêmeas - os jovens foram representados por $3,4 \%$ dos indivíduos, os pequenos adultos por $42,4 \%$ e os grandes adultos por $54,2 \%$. Considerando ambos os sexos, $94,0 \%$ dos peixes encardumados tinham de 16 a $21 \mathrm{~cm}$ de comprimento total. Como as amostras foram retiradas de cardumes capturados pela pesca comercial, é possível que tenha havido alguma seleção pelo tamanho das malhas das redes.

Observando-se a tabela IV, verifica-se que há predominância de machos nas classes de $17-20 \mathrm{~cm}$ de comprimento total, o contrário acontecendo nas classes de maiores tamanhos, o que confirma dados previamente publicados (RossIWONGTSCHOWSKI 1977; MATSUURA 1989). Os valores de K tendem a decrescer a partir da classe de $17 \mathrm{~cm}$ de comprimento total; o decréscimo dos valores $\mathrm{K}$ nas classes de maiores tamanhos das fêmeas tende para 8,5 - valor registrado por VAZZOLER \& VAZZOLER (1965), em fêmeas ovadas no período de reprodução.

As amostras de cardumes da sardinha-verdadeira, distribuídas por classes de tamanho e estações do ano (Tab. V), mostram grandes concentrações de peixes em algumas classes, como se indica para ambos os sexos: outono - sete classes (16-22 $\mathrm{cm})=95,3 \%$; inverno - seis classes $(17-22 \mathrm{~cm})=92,4 \%$; primavera - cinco classes $(17-21 \mathrm{~cm})=96,6 \%$; verão - seis classes $(16-21 \mathrm{~cm})=96,9 \%$. O menor número de 
classes correspondeu à primavera, quando começa o grande período anual de reprodução; as maiores porcentagens de peixes concentrados foram observadas na primavera-verão, que é o grande período anual de reprodução, e as menores foram as do inverno. Há uma relação entre tais parâmetros e a reprodução da sardinha-verdadeira.

Tabela IV. Composição das amostras de cardumes da sardinha-verdadeira, por classes de comprimento total e sexos, com os respectivos valores de $\mathrm{K}$ e relações sexuais. Cardumes capturados pela pesca comercial na área de Angra dos Reis (Rio de Janeiro, Brasil), entre o outono/1999 e o verão/2000.

\begin{tabular}{|c|c|c|c|c|c|c|}
\hline \multirow{2}{*}{$\begin{array}{l}\text { Comprimento total } \\
\qquad(\mathrm{cm})\end{array}$} & \multicolumn{3}{|c|}{ Peixes (\%) } & \multicolumn{2}{|c|}{ Valores de $K(\bar{x} \pm s)$} & \multirow{2}{*}{$\begin{array}{c}\text { Proporção } \\
\text { (machos:fêmeas) }\end{array}$} \\
\hline & $\begin{array}{l}\text { Machos } \\
(n=953)\end{array}$ & $\begin{array}{c}\text { Fêmeas } \\
(n=1.049)\end{array}$ & $\begin{array}{c}\text { Ambos } \\
(n=2.278)\end{array}$ & Machos & Fêmeas & \\
\hline 14 & - & 0,4 & 0,2 & - & $8,0 \pm 0,3$ & - \\
\hline 15 & 0,0 & 0,8 & 1,5 & $9,6 \pm 0,6$ & $8,4 \pm 1,1$ & - \\
\hline 16 & 1,3 & 2,2 & 7,5 & $9,9 \pm 0,9$ & $9,5 \pm 1,3$ & $1,0: 1,1$ \\
\hline 17 & 6,5 & 10,9 & 14,5 & $9,9 \pm 1,0$ & $9,6 \pm 1,3$ & $1,0: 0,8$ \\
\hline 18 & 16,6 & 14,4 & 15,2 & $9,3 \pm 1,0$ & $9,5 \pm 1,2$ & $1,0: 0,7$ \\
\hline 19 & 24,6 & 17,1 & 19,0 & $8,9 \pm 0,7$ & $9,1 \pm 0,9$ & $1,0: 0,8$ \\
\hline 20 & 30,2 & 26,0 & 24,3 & $8,8 \pm 0,6$ & $8,9 \pm 0,6$ & $1,0: 0,9$ \\
\hline 21 & 17,4 & 20,5 & 13,5 & $8,6 \pm 0,6$ & $8,8 \pm 0,5$ & $1,0: 1,6$ \\
\hline 22 & 3,1 & 6,5 & 3,7 & $8,3 \pm 0,6$ & $8,7 \pm 0,6$ & $1,0: 4,4$ \\
\hline 23 & 0,2 & 1,1 & 0,6 & 8,0 & $8,6 \pm 0,8$ & - \\
\hline 24 & - & - & - & - & - & - \\
\hline 25 & - & - & - & - & - & - \\
\hline 26 & 0,1 & - & 0,0 & 3,1 & - & - \\
\hline 27 & - & - & - & - & - & - \\
\hline 28 & - & 0,1 & 0,0 & - & 2,8 & - \\
\hline Total & 100,0 & 100,0 & 100,0 & $9,2 \pm 0,9$ & $9,1 \pm 0,9$ & $1,0: 0,9$ \\
\hline
\end{tabular}

Tabela V. Composição das amostras de cardumes da sardinha-verdadeira por classes de comprimento total, estações do ano e sexos. Cardumes capturados pela pesca comercial na área de Angra dos Reis (Rio de Janeiro, Brasil), entre o outono/1999 e o verão/2000.

\begin{tabular}{|c|c|c|c|c|c|c|c|c|c|c|c|c|}
\hline \multirow{3}{*}{$\begin{array}{c}\text { Comprimento } \\
\text { total }\end{array}$} & \multicolumn{12}{|c|}{ Peixes $(\%)$} \\
\hline & \multicolumn{3}{|c|}{ Outono } & \multicolumn{3}{|c|}{ Inverno } & \multicolumn{3}{|c|}{ Primavera } & \multicolumn{3}{|c|}{ Verão } \\
\hline & $\begin{array}{l}\text { Machos } \\
n=191\end{array}$ & $\begin{array}{l}\text { Fêmeas } \\
n=169\end{array}$ & $\begin{array}{l}\text { Ambos } \\
n=594\end{array}$ & $\begin{array}{l}\text { Machos } \\
n=240\end{array}$ & $\begin{array}{l}\text { Fêmeas } \\
n=331\end{array}$ & $\begin{array}{l}\text { Ambos } \\
n=611\end{array}$ & $\begin{array}{l}\text { Machos } \\
n=291\end{array}$ & $\begin{array}{l}\text { Fêmeas } \\
n=310\end{array}$ & $\begin{array}{l}\text { Ambos } \\
n=602\end{array}$ & $\begin{array}{l}\text { Machos } \\
n=231\end{array}$ & $\begin{array}{l}\text { Fêmeas } \\
n=239\end{array}$ & $\begin{array}{l}\text { Ambos } \\
n=471\end{array}$ \\
\hline 14 & - & - & 0,3 & - & 1,2 & 0,8 & - & - & - & - & - & - \\
\hline 15 & 0,0 & 0,0 & 3,7 & - & 1,8 & 2,0 & 0,3 & - & 0,2 & 0,9 & 0,8 & 0,8 \\
\hline 16 & 2,1 & 0,0 & 17,0 & 2,1 & 3,3 & 3,8 & 2,1 & - & 1,0 & 17,3 & 5,0 & 11,1 \\
\hline 17 & 2,1 & 1,8 & 16,2 & 5,0 & 10,8 & 9,6 & 19,5 & 9,7 & 14,4 & 20,3 & 19,3 & 20,0 \\
\hline 18 & 12,0 & 0,6 & 6,1 & 12,9 & 11,5 & 12,8 & 32,9 & 25,5 & 29,1 & 11,3 & 13,4 & 12,3 \\
\hline 19 & 23,0 & 5,9 & 9,6 & 28,3 & 18,4 & 21,8 & 29,5 & 28,1 & 28,7 & 18,6 & 8,8 & 13,6 \\
\hline 20 & 39,8 & 36,1 & 23,9 & 35,0 & 24,1 & 26,8 & 11,7 & 23,2 & 17,6 & 28,6 & 26,8 & 27,6 \\
\hline 21 & 19,4 & 37,3 & 17,5 & 12,1 & 20,5 & 16,0 & 3,4 & 10,0 & 6.8 & 3,0 & 21,3 & 12,3 \\
\hline 22 & 1,6 & 16,0 & 5,0 & 4,2 & 6,9 & 5,4 & 0,3 & 2,2 & 1,3 & - & 4,6 & 2,3 \\
\hline 23 & - & 2,4 & 0,7 & 0,4 & 1,2 & 0,8 & - & 1,3 & 0,7 & - & - & - \\
\hline 24 & - & - & - & - & - & - & - & - & - & - & - & - \\
\hline 25 & - & - & - & - & - & - & - & - & - & - & - & - \\
\hline 26 & - & - & - & - & - & - & 0,3 & - & 0,2 & - & - & - \\
\hline 27 & - & - & - & - & - & - & - & - & - & - & - & - \\
\hline 28 & - & - & - & - & 0,3 & 0,2 & - & - & - & - & - & - \\
\hline Total & 100,0 & 100,0 & 100,0 & 100,0 & 100,0 & 100,0 & 100,0 & 100,0 & 100,0 & 100,0 & 100,0 & 100,0 \\
\hline
\end{tabular}


Os dados deste trabalho indicam que os cardumes da sardinha-verdadeira, capturados na área de Angra dos Reis, podem ser considerados como cardumes de desova, segundo critérios de NIKOLSKY (1962). Eles ocorrem em área de desova, sendo constituídos quase que exclusivamente por adultos (Tab. VI), havendo um longo período anual de reprodução.

Tabela VI. Ocorrência de agrupamentos do ciclo vital em cardumes da sardinha-verdadeira, por sexos e estações do ano. Cardumes capturados pela pesca comercial na área de Angra dos Reis (Rio de Janeiro, Brasil), entre o outono/1999 e o verão/2000.

\begin{tabular}{|c|c|c|c|c|c|c|c|c|}
\hline \multirow[t]{3}{*}{ Agrupamentos } & \multicolumn{8}{|c|}{ Freqüências nas amostras (\%) } \\
\hline & \multicolumn{2}{|c|}{ Outono } & \multicolumn{2}{|c|}{ Inverno } & \multicolumn{2}{|c|}{ Primavera } & \multicolumn{2}{|c|}{ Verăo } \\
\hline & $\begin{array}{l}\text { Machos } \\
(n=191)\end{array}$ & $\begin{array}{l}\text { Fêmeas } \\
(n=169)\end{array}$ & $\begin{array}{l}\text { Machos } \\
(n=240)\end{array}$ & $\begin{array}{l}\text { Fêmeas } \\
(n=331)\end{array}$ & $\begin{array}{l}\text { Machos } \\
(n=291)\end{array}$ & $\begin{array}{l}\text { Fêmeas } \\
(n=310)\end{array}$ & $\begin{array}{l}\text { Machos } \\
(n=231)\end{array}$ & $\begin{array}{l}\text { Fêmeas } \\
(n=239)\end{array}$ \\
\hline Jovens & 0,0 & 4,3 & - & 6.3 & 0,3 & - & 0.9 & 11.9 \\
\hline Adultos & 100,0 & 95,7 & 100,0 & 93,7 & 99,7 & 100,0 & 99,1 & 88,1 \\
\hline Adultos pequenos & 16,2 & 12,2 & 20,0 & 40,7 & 54,5 & 63,3 & 48,9 & 45,9 \\
\hline Adultos grandes & 83,8 & 83,5 & 80,00 & 53,0 & 45,2 & 36,7 & 50,2 & 42,2 \\
\hline Total & 100,0 & 100,0 & 100,0 & 100,0 & 100,0 & 100,0 & 100,0 & 100,0 \\
\hline
\end{tabular}

O recrutamento ocorre no inverno, dada a baixa freqüência de indivíduos maduros, as menores médias estacionais do comprimento e do peso totais, os pequenos valores de $\mathrm{K}$ e a predominância de fêmeas.

Não foram observadas diferenças sexuais entre as classes de comprimento total e as respectivas médias de peso total (Tab. VII), confirmando que não existe dimorfismo sexual na sardinha-verdadeira (ROSSI-WONGTSCHOWSKI 1977).

Tabela VII. Pesos totais médios $(\bar{x} \pm s)$ da sardinha-verdadeira, em amostras de cardumes, por classes de comprimento total e sexos. Cardumes capturados pela pesca comercial na área de Angra dos Reis (Rio de Janeiro, Brasil), entre o outono/1999 e o verão/2000.

\begin{tabular}{|c|c|c|c|c|c|c|}
\hline \multirow{2}{*}{$\begin{array}{l}\text { Comprimento total } \\
(\mathrm{cm})\end{array}$} & \multicolumn{2}{|c|}{ Machos } & \multicolumn{2}{|c|}{ Fêmeas } & \multicolumn{2}{|r|}{ Ambos } \\
\hline & Peixes (n) & Peso (g) & Peixes (n) & Peso (g) $(\bar{x} \pm s)$ & Peixes (n) & Peso $(g)(\bar{x} \pm s)$ \\
\hline 14 & - & - & 4 & $22,0 \pm 0,3$ & 7 & $23,8 \pm 3,5$ \\
\hline 15 & 4 & $32,9 \pm 5,0$ & 8 & $29,5 \pm 4,5$ & 39 & $32,6 \pm 3,7$ \\
\hline 16 & 54 & $42,7 \pm 5,6$ & 23 & $39,8 \pm 6,9$ & 182 & $41,2 \pm 5,0$ \\
\hline 17 & 120 & $50,1 \pm 5,8$ & 114 & $48,2 \pm 6,6$ & 336 & $48,7 \pm 5,8$ \\
\hline 18 & 176 & $55,3 \pm 6,0$ & 151 & $56,1 \pm 7,1$ & 347 & $55,5 \pm 6,5$ \\
\hline 19 & 241 & $62,5 \pm 5,5$ & 179 & $63,9 \pm 6,8$ & 427 & $63,0 \pm 6,2$ \\
\hline 20 & 259 & $71,0 \pm 5,0$ & 274 & $71,9 \pm 5,7$ & 542 & $71,4 \pm 5,4$ \\
\hline 21 & 83 & $78,4 \pm 5,8$ & 215 & $81,4 \pm 5,9$ & 301 & $80,5 \pm 6,0$ \\
\hline 22 & 14 & $87,0 \pm 7,8$ & 68 & $91,1 \pm 6,6$ & 82 & $90,4 \pm 6,9$ \\
\hline 23 & 1 & 100,9 & 12 & $103,6 \pm 10,6$ & 13 & $103,4 \pm 10,2$ \\
\hline 24 & - & - & - & $112,7 \pm 9,6$ & - & - \\
\hline 25 & - & - & - & - & - & - \\
\hline 26 & 1 & 178,7 & - & - & 1 & 178,7 \\
\hline 27 & - & - & - & - & - & - \\
\hline 28 & - & - & 1 & 176,9 & 1 & 176,9 \\
\hline Total & 953 & $62,5 \pm 11,8$ & 1.049 & $68,0 \pm 15,3$ & 2.278 & $62,915,1$ \\
\hline
\end{tabular}

Observação: há uma grande dispersão de valores nas classes maiores de comprimento total, o que confirma resultado obtido por ROSSI-WONGTSCHOWSKI (1977). 


\section{CONCLUSÕES}

Os machos tiveram médias de comprimento e peso totais menores do que as das fêmeas, com maiores diferenças no peso. As flutuações estacionais das médias do peso total estão associadas ao ciclo anual de reprodução e ao fator de condição, principalmente nas fêmeas. A relação sexual manteve-se em torno de 1:1, exceto no inverno, quando as fêmeas foram a maioria. Foram encontrados machos e fêmeas maduros durante todo o ano, com máximo na primavera-verão, o que mostra um longo período de reprodução, com sucessivas desovas de reprodutores de um mesmo cardume, pois a desova individual é total. Os comprimentos totais em que 50\% dos peixes estão maduros são diferentes para machos $(18,2 \mathrm{~cm})$ e fêmeas $(19,2 \mathrm{~cm})$. Os cardumes podem ser considerados como cardumes de desova, por se encontrarem em área de desova, quase totalmente constituídos por adultos, com longo período anual de reprodução.

AGRADECIMENTOS. Os autores agradecem o apoio recebido da empresa Castro Indústria e Comércio de Pescados Ltda. (Angra dos Reis - RJ - Brasil), na pessoa do seu diretor-presidente Isake de Castro.

\section{REFERÊNCIAS BIBLIOGRÁFICAS}

ISAAC-NAhUM, V.J.; A.E.A.M. VAZZOLER \& E.M. ZANETTI-PRADO. 1983. Estudos sobre estrutura, ciclo de vida e comportamento de Sardinella brasiliensis (Steindachner, 1879), na área entre $22^{\circ} \mathrm{S}$ e $28^{\circ}$ S, Brasil. 3. Morfologia e histologia de ovários e escala de maturidade. Bol. Inst. oceanogr., São Paulo, 32 (1): 1-16.

Matsuura, Y. 1977. O ciclo de vida da sardinha-verdadeira (Introdução à oceanografia pesqueira). Publ. Esp. Inst. oceanogr., São Paulo, (4): 1-146.

. 1989. Synopsis on the reproductive and early life history of the Brazilian sardine, Sardinella brasiliensis, and related environmental conditions. IOC Workshop Report, Montevideo, (65): 1-8.

Moyle, P.B. \& J.J. CeCH JR. 1982. Fishes: an introduction to ichthyology. Englewood Cliffs, Prentice-Hall, XIV+593p.

Nikolsky, G.V. 1962 (1978). The Ecology of Fishes. Neptune City, T.F.H. Publications, 352p.

PaIVA, M.P. 1997. Recursos pesqueiros estuarinos e marinhos do Brasil. Fortaleza, UFC Edições, $286 \mathrm{p}$.

PAIVA, M.P. \& P.C.S. MotTA. 2000. Cardumes da sardinha-verdadeira, Sardinella brasiliensis (Steindachner), em águas costeiras do estado do Rio de Janeiro, Brasil. Revta bras. Zool. 17 (2): 339-346.

RosSI-WONGTSCHOWSKI, C.I.B. 1977. Estudo das variações da relação peso/comprimento total em função do ciclo reprodutivo e comportamento de Sardinella brasiliensis (Steindachner, 1879) da costa do Brasil entre $23^{\circ} \mathrm{S}$ e $28^{\circ} \mathrm{S}$. Bol Inst. Oceanogr., São Paulo, 26: 131-180.

VAlEntini, H. \& R.D. CARdoso. 1991. Análise da pesca da sardinha-verdadeira, Sardinella brasiliensis, na costa sudeste-sul do Brasil. Atlântica, Rio Grande, 13 (1): 45-54.

VAZZOLER, A.E.A.M. \& C.L.D.B. Rossi-WONGTSCHOWSKI. 1976. Sardinella brasiliensis: tipo de desova, fecundidade e potencial reprodutivo. I. Área entre $23^{\circ} 40^{\prime}$ S e $24^{\circ} 20^{\prime}$ S, Brasil. Bolm Inst. oceanogr., São Paulo, 25: 131-155.

VAZZOLER, A.E.A.M. \& G. VAZZOLER. 1965. Relation between condition factor and sexual development in Sardinella aurita (Cuv. \& Val., 1847). An. Acad. bras. Ciênc. 37 (Supl.): 353-359.

Recebido em 24.VI.2002; aceito em 08.XI.2002. 